\title{
OPTIMIZATION AND SIMULATION FOR AIRPORT EMERGENCY INVENTORY WITH REPLACEMENT
}

\author{
Meng, Q. C. ; Guo, Y. ; Zhao, P. X. ${ }^{*, \# ; ~ L u, ~ T . ~ X . * ; ~ W a n, ~ X . ~ L . ~}{ }^{*}$; Rong, X. X. ${ }^{* *}$ \& Pan, W. ${ }^{* * *}$ \\ *School of Management, Shandong University, Jinan 250100, China \\ ${ }^{* *}$ School of Mathematics, Shandong University, Jinan 250100, China \\ *** School of Economics and Management, Wuhan University, Wuhan 430072, China \\ E-Mail: pxzhao@sdu.edu.cn ( ${ }^{\#}$ Corresponding author)
}

\begin{abstract}
The paper assumes that the accident occurrence time is stochastic and emergency supplies is perishable, then two replacement stochastic models based on remaining lifetime and remaining quantity are first proposed. In order to identify the effectiveness of replacement strategy, two replacement-based stochastic models are compared with the general stochastic model that is non-replacement, measured by inventory level and total costs. A discrete-event simulation model is developed to demonstrate effects of occurrence time uncertainty, replacement ratios and distributed functions in occurrence time and demand. Sensitive analysis shows that the optimal decision is more sensitive to remaining quantity ratio as compared to remaining lifetime ratio. The paper shows that when decision-makers ignore occurrence time uncertainty and limited warehousing time, they may significantly miss better decisions. Further, simulation results demonstrate that different distributed functions in both occurrence time and demand lead to different inventory strategies.

(Received, processed and accepted by the Chinese Representative Office.)
\end{abstract}

Key Words: Occurrence Time Uncertainty, Emergency Supplies, Replacement strategy, Inventory Optimization

\section{INTRODUCTION}

Although civil aviation accident is event of small probability, the losses and fatalities are serious. For example, there were 92 accidents and 474 fatalities last year, which leads to big losses for companies and society. According to safety report from International Civil Aviation Organization (ICAO), about $70 \%$ civil aviation accidents occurred around the airports, so the ICAO formulated a series of regulations for emergency supplies in the airport. The airports always reserve much more emergency supplies and replenish emergency supplies immediately after emergency response. Therefore, investments in airport emergency supplies are significant and a cause of financial concern and subsequent economic losses. Thus, minimizing the losses and the costs, while maintaining the constraint of service level, is the main purpose of the airport.

In this paper, we first propose replacement strategy and develop an integrated model of occurrence time uncertainty and limited warehousing time, capturing the effects of occurrence time uncertainty and replacement strategy on service level and total costs. Our designs of alternative inventory system are derived from two sources: (1) observations of emerging practices in returns processing and (2) previous researches on emergency supplies inventory management. We analyse a benchmark system where an airport emergency planner decides the inventory level with replacement strategy to minimize the total costs. We find that the inventory level in this benchmark system is not always higher or less than that in non-replacement setting. To understand how occurrence time uncertainty affects the inventory level, we setup the model with deterministic occurrence time and unlimited warehousing time, concluding that consideration of occurrence time uncertainty and limited warehousing time induces or reduces emergency inventory. 
Researches on emergency supplies management obtain many achievements in preposition, optimal inventory, planning and distribution [1-4], and help reduce losses of life and property in practice [5-8]. Emergency means uncertainty, so stochastic models, with stochastic lead time, demand, supply, procurement cost, transport cost etc., were proposed to fulfil purposes of minimizing cost and maximizing satisfaction degree of people in affected areas [9-10]. Paper [11] set up a three-level model containing supply, storage and demand for the emergency planning at the preparation and response stage, and the objective of this model is minimizing the social cost. Research [12] proposed a coordination system among emergency warehouses. In this system, a stochastic model was built with constraints of service egality, traffic congestion and response time. In [13], authors established a stochastic model to decide optimal storage time. Pan et al. [14] proposed a stochastic inventory model with stochastic occurrence time, stochastic demand and perishable emergency supplies.

Civil aviation emergency decision is a prospective issue, but return and replacement of emergency supplies is insignificant, except for commercial products [15]. In [16], authors came up with a novel reverse logistics system for post-disaster debris capable of systematically minimizing total reverse logistical costs, risk-induced cost and psychological cost. The effectiveness of the proposed system was demonstrated by applying it to a case study of Wenchuan earthquake. Paper [17] proposed an effective location models for sorting recyclables in public management, and provided an optimal location planning design for recycling urban solid wastes given the uncertainty future outcome of economic factors, consumer behaviour and environmental awareness. Paper [18] described an exploratory study of reverse exchange systems used for medical devices in the UK National Health Service.

Emergency management for civil aviation mainly includes designing information systems, simulation of emergency evacuation, and formulating emergence planning. Paper [19] analysed Singapore Airlines Flight 006 aircraft accident in 2000, and suggested that plan, execute, and support systems are important for medical emergency supplies in airport. Pan and Guo [20] studied the compensation mechanism based on bargaining game strategy on the background of MH370. In [21], authors proposed an accident rescue model for railroad system, and considered the uncertainty of demand, idle probability of ambulance, and risk level in a section of railway. Similarly to railway rescue system, the airport rescue systems are couple with uncertainty. ICAO stipulates that the airport should reserve emergency supplies to enhance response capability. The emergency supplies include alcohol, blood, plasma and some other perishable medical materials [22]. Inventory management of perishable products also has attracted much attention [23-25].

Emergency decisions for disasters need to be capable of adapting to random and highly dynamic change [26], and decision-makers have a growing interest in improved strategies from reverse logistics [27]. Thus, a significant difference between our model and previous research on emergency supplies management is that we introduce the replacement strategy facing occurrence time uncertainty into the inventory control system. The optimal inventory levels and total costs of models are compared to show the effects of the replacement strategy and the occurrence time uncertainty. The deficient research about return of emergency supplies and the important practice of emergency inventory make our work valuable.

This paper is organized as follows. In section 2, the researched problem is defined and described. Replacement model based on remaining time and remaining quantity are presented with unknown stochastic distribution functions in section 3. In section 4, we compare inventory level and total costs of different models and present analyses about effectiveness of replacement strategy and occurrence time uncertainty. In section 5, we develop a discrete-event simulation model to demonstrate and analyse decisions, applying the results to some simple numerical cases. Finally, conclusions are reached in section 6. 


\section{PROBLEM DESCRIPTION}

In practice, emergency supplies for civil aviation accidents generally come from airport's inventory and some specified rescue units. The emergency supplies ordering system is different from quantitative order method or regular order method. The ending time of a single emergency phase is $\min (t, T)$, among which $t$ represents stochastic civil aviation occurrence time and $T$ represents shelf life of emergency supplies. In other words, the preparation phase will enter into a new circulate phase when emergency response is end or emergency supplies are expired. We assume the stochastic demand and the stochastic occurrence time are mutually independent.

For the purpose of our analysis, the following notations are used:

$e-$ the unit expired cost of emergency supplies,

$s \quad$ - the unit shortage cost of emergency supplies,

$k-$ the unit exchange or replacement cost of emergency supplies,

$\theta$ - the ratio of expected expired quantity to remaining quantity, $0 \leq \theta \leq 1$,

$x$ - the stochastic demand of emergency supplies at stage of emergency response,

I - the optimal inventory level, a decision variable,

$t$ - stochastic civil aviation occurrence time,

$T-$ shelf life of emergency supplies,

$a, b$ - the lower and upper bound of stochastic occurrence time $t$,

$c, d$ - the lower and upper bound of stochastic demand $x$,

$q_{1}$ - the replacement lifetime ratio, defined by time at replacement dividing lifetime,

$q_{2}$ - the replacement quantity ratio, defined by amount of usage dividing total inventory,

$f(x)$ - probability density function of stochastic demand,

$g(t)$ - probability density function of stochastic occurrence time,

$F(x)$ - cumulative density function of stochastic demand,

$G(t)$ - cumulative density function of stochastic occurrence time,

$(A)^{+}-\max (0, A)$, that is, $(A)^{+}=\max (0, A)$.

The two replacement scenarios are proposed based on checking system of emergency supplies in airport practically. At the end of emergency phase, airport will check inventory. If the remaining lifetime is short or remaining quantity is large, in order to avoid the huge expired losses, airport will ask suppliers to return and exchange the surplus materials.

Scenario 1: airport and suppliers reach an agreement on replacement lifetime ratio $q_{1}$, (1) if civil aviation accident occurs within $q_{1} T$, that is, when the remaining life time is longer than $\left(1-q_{1}\right) T$, new emergency supplies will be replenished to the inventory level $I$ without replacement strategy; (2) if civil aviation accident occurs beyond $q_{1} T$, and the remaining life time is shorter than $\left(1-q_{1}\right) T$, all surplus materials will be replaced by suppliers and amount $I$ of new emergency supplies will be transported to airport; (3) if no civil aviation accident occurs within shelf life $T$, all emergency supplies will be expired and airport affords the expired losses. Scenario 1 is the replacement strategy based on remaining lifetime.

Scenario 2: airport and suppliers reach an agreement on replacement quantity ratio $q_{2}$, (1) if civil aviation accident demand is less than the amount $q_{2} I$, in other words, when the remaining quantity is larger than $\left(1-q_{2}\right) I$, all surplus materials will be replaced by suppliers and amount $I$ of new emergency supplies will be transported to airport; (2) if civil aviation accident demand exceeds the amount $q_{2} I$, and the remaining quantity is smaller than $\left(1-q_{2}\right) I$, new emergency supplies will be replenished to the inventory level $I$ without replacement strategy; (3) if no civil aviation accident occurs within shelf life $T$, all emergency supplies will be expired and airport affords the expired losses. Scenario 2 is the replacement strategy based on remaining quantity. 
Inventory level represents service level, and the effectiveness of the replacement-based strategy can be measured by losses and inventory level through building the model that is non-replacement. The effects of occurrence time uncertainty on inventory level are analysed by building the model with deterministic occurrence time and unlimited warehousing time. Subscript 1, 2, 3 represents the replacement model based on remaining lifetime, replacement model based on remaining quantity, and general model without replacement respectively.

\section{MODELS WHEN REPLACEMENT IS ALLOWED}

\subsection{Replacement model based on remaining lifetime}

The expected losses include shortage losses, possible expired losses, replacement costs, and expired losses for total emergency supplies.

$$
\begin{aligned}
& E T C_{1}=\int_{a}^{T} \int_{I}^{d} s(x-I) f(x) g(t) d x d t+\int_{a}^{q_{1} T} \int_{c}^{I} \theta e(I-x) f(x) g(t) d x d t \\
& +\int_{q_{1} T}^{T} \int_{c}^{I} k(I-x) f(x) g(t) d x d t+\int_{T}^{b} \int_{c}^{d} e I f(x) g(t) d x d t \\
& d_{E T C_{1}} / d I=-\int_{a}^{T} \int_{I}^{d} s f(x) g(t) d x d t+\int_{a}^{q_{1} T} \int_{c}^{I} \theta e f(x) g(t) d x d t \\
& +\int_{q_{1} T}^{T} \int_{c}^{I} k f(x) g(t) d x d t+\int_{T}^{b} \int_{c}^{d} e f(x) g(t) d x d t \\
& d^{2} E T C_{1} / d I^{2}=s f(I) G(T)+\theta e f(I) G(T)+k f(I)\left(G(T)-G\left(q_{1} T\right)\right)
\end{aligned}
$$

Eq. (3) is greater than zero, and the unique solution of Eq. (1) can be reached by letting Eq. (2) being equal to zero.

$$
I_{1}^{*}=F^{-1}\left(\left(\frac{(s+e) G(T)-e}{(s+k) G(T)+(\theta e-k) G\left(q_{1} T\right)}\right)^{+}\right)
$$

Proposition 1: $\frac{\partial I_{1}^{*}}{\partial s} \geq 0, \frac{\partial I_{1}^{*}}{\partial e} \leq 0, \frac{\partial I_{1}^{*}}{\partial \theta} \leq 0, \frac{\partial I_{1}^{*}}{\partial k} \leq 0$.

Proposition 1 shows that the optimal inventory level increases with the increase of unit shortage cost, and decreases with the increase of unit expired cost, expired rate and unit replacement cost. Furthermore, the optimal inventory level may increase or decrease with the increase of lifetime, and it depends on the distribution function of stochastic occurrence time.

\subsection{Replacement model based on remaining quantity}

$$
\begin{aligned}
\text { ETC }_{2}= & \int_{a}^{T} \int_{I}^{d} s(x-I) f(x) g(t) d x d t+\int_{a}^{T} \int_{q_{2} I}^{I} \theta e(I-x) f(x) g(t) d x d t \\
& +\int_{a}^{T} \int_{c}^{q_{2} I} k(I-x) f(x) g(t) d x d t+\int_{T}^{b} \int_{c}^{d} e I f(x) g(t) d x d t
\end{aligned}
$$

Proposition 2: The existence of solution of Eq. (4) is dependent on the distributed functions in stochastic demand. If the solution $I_{2}{ }^{*}$ can be reached, then the following holds:

$$
\begin{aligned}
& s F\left(I_{2}^{*}\right) G(T)+\theta e F\left(I_{2}^{*}\right) G(T)-\theta e F\left(q_{2} I_{2}^{*}\right) G(T)-q_{2} \theta e\left(I_{2}^{*}-q_{2} I_{2}^{*}\right) f\left(q_{2} I_{2}^{*}\right) G(T) \\
& +k F\left(q_{2} I_{2}^{*}\right) G(T)+q_{2} k\left(I_{2}^{*}-q_{2} I_{2}^{*}\right) f\left(q_{2} I_{2}^{*}\right) G(T)+e-e G(T)-s G(T)=0
\end{aligned}
$$

Proposition 3: The effects of parameters (shortage cost, expired cost, replacement cost, replacement quantity ratio, expected expiration rate etc.) on optimal inventory level depend on the distribution function of stochastic demand.

From Proposition 2 and Proposition 3, the existence of optimal solution and the effects of parameters is correlated to the distributed function in stochastic demand, and to but much less to the distributed function in stochastic occurrence time. The possible explanation is that the stochastic occurrence time cannot affect the integrand in this construction function. 


\section{ANALYSIS FOR REPLACEMENT STRATEGY}

To demonstrate the effectiveness of replacement-based strategy, the general storage model with non-replacement strategy is proposed at the same time.

\subsection{General model with non-replacement}

$$
E T C_{3}=\int_{a}^{T} \int_{I}^{d} s(x-I) f(x) g(t) d x d t+\int_{a}^{T} \int_{c}^{I} \theta e(I-x) f(x) g(t) d x d t+\int_{T}^{b} \int_{c}^{d} e I f(x) g(t) d x d t
$$

The second-order derivative is greater than zero, and the unique optimal solution of Eq. (7) can be calculated through letting first-order derivative being equal to zero.

$$
I_{3}^{*}=F^{-1}\left(\left(\frac{(s+e) G(T)-e}{(s+\theta e) G(T)}\right)^{+}\right)
$$

Proposition 4: $\frac{\partial I_{3}^{*}}{\partial T} \geq 0, \frac{\partial I_{3}^{*}}{\partial s} \geq 0, \frac{\partial I_{3}^{*}}{\partial e} \leq 0, \frac{\partial I_{3}^{*}}{\partial \theta} \leq 0$.

Proposition 4 shows that the optimal inventory level of Eq. (5) increases with the increase of the shelf life and unit shortage cost, and decreases with the increase of unit expired cost and possible expired rate.

\subsection{Comparison and analysis}

Considering replacement strategy complicates the inventory management problem and raises new issues when determining the optimal inventory strategies. To provide closed-form expressions, we assume both stochastic demand and stochastic occurrence time conform to a specific distribution. In order to testify the different effects of different distribution on the optimal inventory strategies, the different distributed functions in stochastic variable are extended in and numerical simulation section.

Three popular stochastic distribution types are uniform distribution, normal distribution, and exponential distribution. Normal distribution requires a large amount of history data to define its parameters [9]. Exponential distribution seems to demonstrate the fundamental characteristics of random occurrence time [28], and it also requires mutually independent data to define parameters. However, for civil aviation accidents, the historical data are often sparse, and accidents are not mutually independent because of ex post measures [29]. In addition, the real data in paper [30] reveals that occurrence time conforms to uniform distribution in practice. Therefore, assumptions of uniform distributed function in stochastic occurrence time and uniformed distributed function in stochastic demand are reasonable and feasible. Then the solutions of Eqs. (1), (4), and (5) are obtained.

$$
\begin{aligned}
& I_{1}^{*}=\left(\frac{(s+e) G(T)-e}{(s+k) G(T)+(\theta e-k) G\left(q_{1} T\right)}\right)^{+} \cdot(d-c)+c \\
& I_{2}^{*}=\max \left(\frac{(s+k)(T-a) c-e(b-T)(d-c)}{(T-a)\left(2 k q_{2}-k q_{2}{ }^{2}+s+\theta e-2 \theta e q_{2}+\theta e q_{2}^{2}\right)}, c\right) \\
& I_{3}^{*}=\left(\frac{(s+e) G(T)-e}{(s+\theta e) G(T)}\right)^{+}(d-c)+c
\end{aligned}
$$

\subsection{Replacement model based on remaining lifetime versus general model}

Lemma 1: When $\theta e>k$, then $\frac{\partial\left(E T C_{3}-E T C_{1}\right)}{\partial q_{1}}<0$ and $E T C_{3} \geq E T C_{1}$. When $\theta e \leq k$, then $\frac{\partial\left(E T C_{3}-E T C_{1}\right)}{\partial q_{1}} \geq 0$ and $E T C_{3} \leq E T C_{1}$. 
Proof: $\frac{\partial\left(E T C_{3}-E T C_{1}\right)}{\partial q_{1}}=\frac{1}{(b-a)(d-c)} \cdot(\theta e-k)(I-c)\left(\frac{I+c}{2}-I\right)$, and $I \geq c$.

With a larger $q_{1}$, the probability of civil aviation accident occurring within $q_{1} T$ is larger and the remaining lifetime is shorter when asked to replace the surplus materials, leading to the larger probability of being expiration and not replacing surplus materials. Thus, if unit possible expired loss is higher than unit replacement cost, the expected losses will increase with the increase of $q_{1}$. Otherwise, the expected losses ETC $C_{1}$ will decrease with the increase of $q_{1}$ if unit replacement cost is higher.

Lemma 2: When $\theta e>k$, then $I_{3}{ }^{*}<I_{1}{ }^{*}$ and $\partial\left|I_{1}^{*}-I_{3}^{*}\right| / \partial q_{1} \leq 0$. When $\theta e \leq k$, then $I_{3}{ }^{*} \geq I_{1}{ }^{*}$ and $\partial\left|I_{1}^{*}-I_{3}^{*}\right| / \partial q_{1} \leq 0$.

Proof: Due to $1 / I_{3}^{*}-1 / I_{1}^{*}=\frac{(\theta e-k)\left(G(T)-G\left(q_{1} T\right)\right)}{((s+e) G(T)-e)(d-c)}$ and $G(T)-G\left(q_{1} T\right) \geq 0$.

Thus, when $\theta e>k$, then $1 / I_{3}{ }^{*}>1 / I_{1}{ }^{*}$; and when $\theta e \leq k$, then $1 / I_{3}{ }^{*} \leq 1 / I_{1}{ }^{*}$.

In addition, when $\theta e>k$, then:

$$
\frac{\partial\left|I_{1}^{*}-I_{3}^{*}\right|}{\partial q_{1}}=\partial\left(\frac{(s+e) G(T)-e}{(s+k) G(T)+(\theta e-k) G\left(q_{1} T\right)}-\frac{(s+e) G(T)-e}{(s+\theta e) G(T)}\right) / \partial q_{1} \leq 0 .
$$

and when $\theta e \leq k$, then:

$$
\frac{\partial\left|I_{1}^{*}-I_{3}^{*}\right|}{\partial q_{1}}=\partial\left(\frac{(s+e) G(T)-e}{(s+\theta e) G(T)}-\frac{(s+e) G(T)-e}{(s+k) G(T)+(\theta e-k) G\left(q_{1} T\right)}\right) / \partial q_{1} \leq 0
$$

Airport tends to store more emergency supplies to reduce shortage losses if unit replacement cost is lower. Contrarily, the airport tends to store less emergency supplies to reduce the high replacement cost if unit replacement cost is higher than unit possible expired loss. In addition, $q_{1}$ cannot change the relationship between $I_{1}{ }^{*}$ and $I_{3}{ }^{*}$.

Theorem 1: The replacement strategy based on remaining lifetime can improve emergency supplies inventory strategies when $\theta e>k$.

Theorem 1 can be demonstrated by Lemma 1 and Lemma 2. When unit replacement cost is lower than unit possible expired loss, the replacement strategy can reduce the expected losses and enhance the inventory level no matter what the value of $q_{1}$ is. Therefore, the airport should bargain with suppliers to lowing replacement cost, and can benefit from the replacement strategy based on remaining lifetime.

\subsection{Replacement model based on remaining quantity versus general model}

Lemma 3:When $\theta e>k, \frac{\partial\left(E T C_{3}-E T C_{2}\right)}{\partial q_{2}}>0$. When $\theta e \leq k, \frac{\partial\left(E T C_{3}-E T C_{2}\right)}{\partial q_{2}} \leq 0$. There exists a turning point $\tilde{q}_{2}=c / I$, making $\left(E T C_{3}-E T C_{2}\right)_{q_{2}=c / I}=0 \quad\left(\tilde{q}_{2}=2-c / I \geq 1\right.$ is rejected $)$.

Proof: $\frac{\partial\left(E T C_{3}-E T C_{2}\right)}{\partial q_{2}}=\frac{1}{(b-a)(d-c)} \cdot(\theta e-k) I^{2}\left(1-q_{2}\right)(T-a)$, and $1-q_{2} \geq 0, T-a \geq 0$.

For a larger $q_{2}$, the probability of replacing surplus materials is larger and the probability of expiration is smaller. Thus, if unit possible expired loss is higher than unit replacement cost, the expected losses will increase with the increase of $q_{2}$. Otherwise, the expected losses $E T C_{2}$ will decrease with the increase of $q_{2}$. So the value of $q_{2}$ can change the decision whether to select the replacement strategy based on remaining quantity or not. 
Table I: The gap of expected losses.

\begin{tabular}{|c|c|c|}
\hline & $q_{2}>\tilde{q}_{2}$ & $q_{2} \leq \tilde{q}_{2}$ \\
\hline$\theta e>k$ & $E T C_{3}>E T C_{2}$ & $E T C_{3} \leq E T C_{2}$ \\
\hline$\theta e \leq k$ & $E T C_{3} \leq E T C_{2}$ & $E T C_{3}>E T C_{2}$ \\
\hline
\end{tabular}

Lemma 4: There exists a turning point $\bar{q}_{2}$, making $I_{3}{ }^{*} \leq I_{2}{ }^{*}$ when $q_{2} \leq \bar{q}_{2}$, and $I_{3}{ }^{*}>I_{2}{ }^{*}$ when $q_{2}>\bar{q}_{2}$.

Lemma 4 can be illustrated by the closed-form solution of equation $I_{3}{ }^{*}-I_{2}{ }^{*}=0$ and $\frac{\partial\left(I_{3}^{*}-I_{2}^{*}\right)}{\partial q_{2}} \geq 0$. Thus, the value of $q_{2}$ can change the relationship between $I_{2}{ }^{*}$ and $I_{3}{ }^{*}$.

Theorem 2: The effective replacement strategy based on remaining quantity satisfies:

(1) If $\bar{q}_{2}>\tilde{q}_{2}$, when $\theta e>k$, then $q_{2}$ should satisfy $\tilde{q}_{2}<q_{2} \leq \bar{q}_{2}$, and when $\theta e \leq k$, then $q_{2}$ should satisfy $q_{2} \leq \tilde{q}_{2}$.

(2) If $\bar{q}_{2} \leq \tilde{q}_{2}$, when $\theta e \leq k$, then $q_{2}$ should satisfy $q_{2} \leq \bar{q}_{2}$, and when $\theta e>k$, there is no complete effective range.

Theorem 2 can be demonstrated by Lemma 3 and Lemma 4, different from scenario 1, we are not sure the replacement-based strategy must be effective for airport. In order to reduce losses and enhance service level simultaneously, airports should lower the unit replacement cost by coordination or technologies, and control the size of $q_{2}$ at the same time.

\subsection{Further discussions about replacement and occurrence time uncertainty}

In classical newsboy model, a stochastic storage model is proposed with stochastic demand and deterministic occurrence time and unlimited warehousing time (lifetime). Thus, the optimal solutions to the classical newsboy models with replacement based on remaining quantity and with non-replacement are calculated as follows.

$$
\bar{I}_{2}=\max \left(\frac{s d+k c}{2 k q_{2}-k q_{2}{ }^{2}+s+\theta e-2 \theta e q_{2}+\theta e q_{2}^{2}}, c\right), \bar{I}_{3}=F^{-1}\left(\frac{s}{s+\theta e}\right) .
$$

- Effects of the value of ratios on inventory level

Theorem 3: When $\theta e>k, \frac{\partial I_{1}^{*}}{\partial q_{1}} \leq 0, \frac{\partial I_{2}^{*}}{\partial q_{2}}>0$. When $\theta e \leq k, \frac{\partial I_{1}^{*}}{\partial q_{1}}>0, \frac{\partial I_{2}^{*}}{\partial q_{2}} \leq 0$.

The results of Theorem 3 can be illustrated well by the characteristics of replacement strategy based on remaining lifetime and replacement strategy based on remaining quantity.

- Effects of occurrence time uncertainty on inventory level

Theorem 4: $\bar{I}_{2}>I_{2}^{*}, \bar{I}_{3}>I_{3}^{*}$.

Theorem 4 can be obtained by calculating $\bar{I}_{2}-I_{2}{ }^{*}$ and $\bar{I}_{3}-I_{3}{ }^{*}$, and the results show that occurrence time uncertainty leads to lower inventory level. In theory, the optimal solution to the decision variable with stochastic occurrence time should consist with that with deterministic occurrence time under risk-neutral criteria. However, the ending time of a single emergency preparation is assumed $\min (t, T)$, meaning that the cycle time is shortened and the order quantity is reduced accordingly.

\section{SIMULATION RESULTS}

A simulation case is proposed and analysed firstly assuming stochastic occurrence time conforms to uniform distribution from $a$ to $b$ and stochastic demand conforms to uniform distribution from $c$ to $d$. Then we simulate, analyse and compare the optimal inventory level 
when stochastic variables conform to different distributions such as exponential distributions and normal distributions. In the absence of realistic data, we estimate parameters by taking advantage of relative literature $[9,14,20]$. Several mid-size problems are designed.

Table II: Specific value of parameters.

\begin{tabular}{|c|c|c|c|c|c|c|c|c|}
\hline$a$ & $b$ & $c$ & $d$ & $T$ & $s$ & $e$ & $\theta$ & $k$ \\
\hline 1 & 3 & 10 & 110 & 2 & 20 & 12 & 0.5 & 10 \\
\hline
\end{tabular}

From results in Table III and Table IV, we find that considering occurrence time uncertainty and limited warehousing time reduces service level by $4.3 \%$, while reduces total costs by $51 \%$. We also show that the value interval will affect the optimal solutions, as well as distributed functions. Furthermore, the replacement strategy based on remaining lifetime seems to be more reliable facing occurrence time uncertainty and demand uncertainty, resulting from total costs variance between replacement strategy based on remaining lifetime $(0.32 \%-0.37 \%$ in example $1 ; 0.36 \%-2.67 \%$ in example 2$)$ and replacement strategy based on remaining quantity $(-181.54 \%-0.96 \%$ in example $1 ; 12.34 \%$ in example 2$)$. The simulation results and explanations of distribution functions are presented in Figs. 1 to 6.

Table III: Optimal solutions to all models for $c=100$ example.

\begin{tabular}{|c|c|c|c|c|c|c|c|c|c|c|c|}
\hline $\boldsymbol{q}_{\mathbf{1}} \boldsymbol{q}_{\mathbf{2}}$ & $\mathbf{0}$ & $\mathbf{0 . 1}$ & $\mathbf{0 . 2}$ & $\mathbf{0 . 3}$ & $\mathbf{0 . 4}$ & $\mathbf{0 . 5}$ & $\mathbf{0 . 6}$ & $\mathbf{0 . 7}$ & $\mathbf{0 . 8}$ & $\mathbf{0 . 9}$ & $\mathbf{1}$ \\
\hline $\boldsymbol{I}_{\mathbf{1}}{ }^{*}$ & 103.3 & 103.4 & 103.6 & 103.7 & 103.8 & 104 & 104.2 & 104.3 & 104.5 & 104.8 & 105 \\
\hline $\boldsymbol{E T C C}_{\mathbf{1}}{ }^{*}$ & 646.1 & 646 & 646.1 & 646 & 645.9 & 646 & 646.1 & 645.9 & 646 & 646.2 & 646.3 \\
\hline $\boldsymbol{I}_{\mathbf{2}}{ }^{*}$ & 110 & 107.6 & 105 & 102.7 & 100.8 & 100 & 100 & 100 & 100 & 100 & 100 \\
\hline $\boldsymbol{E T C}_{\mathbf{2}}{ }^{*}$ & -525 & -274 & -56.9 & 127.9 & 284.5 & 400 & 490 & 560 & 610 & 640 & 650 \\
\hline $\boldsymbol{I}_{\mathbf{3}}{ }^{*}$ & 103.1 & 103.1 & 103.1 & 103.1 & 103.1 & 103.1 & 103.1 & 103.1 & 103.1 & 103.1 & 103.1 \\
\hline $\boldsymbol{E T C}_{\mathbf{3}}{ }^{*}$ & 643.8 & 643.8 & 643.8 & 643.8 & 643.8 & 643.8 & 643.8 & 643.8 & 643.8 & 643.8 & 643.8 \\
\hline $\boldsymbol{I}_{\mathbf{2}}$ & 123 & 119.6 & 116.6 & 114.1 & 112 & 110.3 & 109 & 108 & 107.2 & 106.8 & 106.7 \\
\hline $\boldsymbol{E T C}_{\mathbf{2}}$ & -1116 & -597 & -159 & 209.6 & 516.8 & 768.4 & 969.3 & 1122 & 1228 & 1292 & 1313 \\
\hline $\boldsymbol{I}_{\mathbf{3}}$ & 107.7 & 107.7 & 107.7 & 107.7 & 107.7 & 107.7 & 107.7 & 107.7 & 107.7 & 107.7 & 107.7 \\
\hline $\boldsymbol{E T C}_{\mathbf{3}}$ & 1316 & 1316 & 1316 & 1316 & 1316 & 1316 & 1316 & 1316 & 1316 & 1316 & 1316 \\
\hline
\end{tabular}

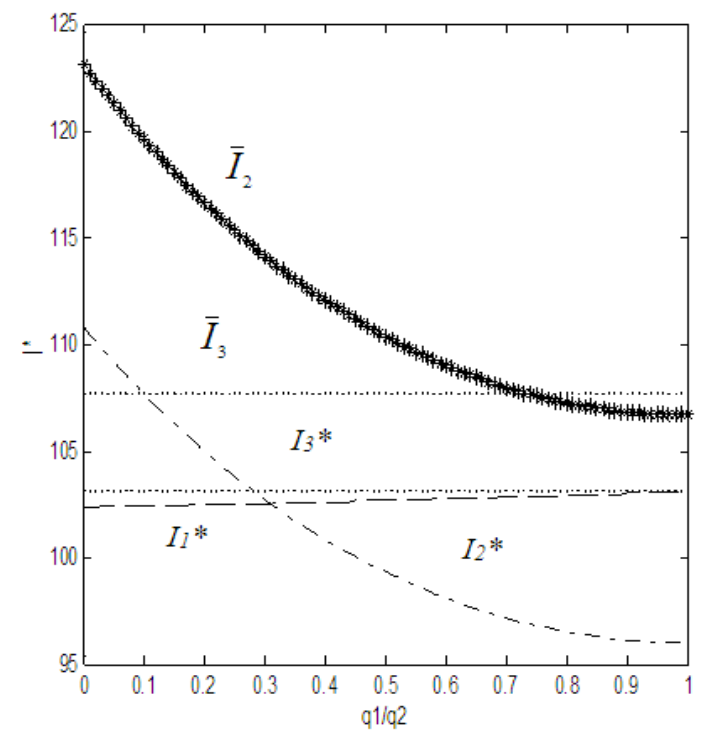

Figure 1: Stochastic occurrence time and stochastic demand conform to uniform distribution.

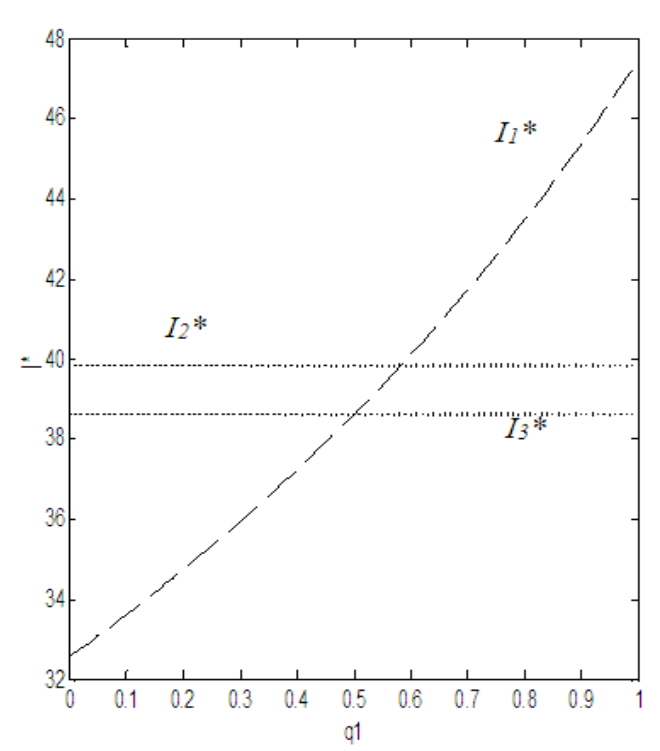

Figure 2: Stochastic occurrence time and stochastic demand conform to uniform distribution and exponential distribution respectively with $\lambda=1 / 105$. 
Table IV: Optimal solutions to all models for $c=10$ example.

\begin{tabular}{|c|c|c|c|c|c|c|c|c|c|}
\hline $\boldsymbol{q}_{\mathbf{1}} / \boldsymbol{q}_{\mathbf{2}}$ & $\mathbf{0 . 1}$ & $\mathbf{0 . 2}$ & $\mathbf{0 . 3}$ & $\mathbf{0 . 4}$ & $\mathbf{0 . 5}$ & $\mathbf{0 . 6}$ & $\mathbf{0 . 7}$ & $\mathbf{0 . 8}$ & $\mathbf{0 . 9}$ \\
\hline $\boldsymbol{I}_{\mathbf{1}}{ }^{*}$ & 34.1 & 34.7 & 35.4 & 36 & 36.7 & 37.4 & 38.2 & 39 & 39.9 \\
\hline $\boldsymbol{E T C}^{*}{ }^{*}$ & 511.8 & 510.6 & 509.4 & 508.1 & 506.7 & 505.2 & 503.7 & 502 & 500.3 \\
\hline $\boldsymbol{I}_{\mathbf{2}}{ }^{*}$ & 10 & 10 & 10 & 10 & 10 & 10 & 10 & 10 & 10 \\
\hline $\boldsymbol{E T C}_{\mathbf{2}}{ }^{*}$ & 560 & 560 & 560 & 560 & 560 & 560 & 560 & 560 & 560 \\
\hline $\boldsymbol{I}_{\mathbf{3}}{ }^{*}$ & 40.8 & 40.8 & 40.8 & 40.8 & 40.8 & 40.8 & 40.8 & 40.8 & 40.8 \\
\hline $\boldsymbol{E T C}_{\mathbf{3}}{ }^{*}$ & 498.5 & 498.5 & 498.5 & 498.5 & 498.5 & 498.5 & 498.5 & 498.5 & 498.5 \\
\hline
\end{tabular}

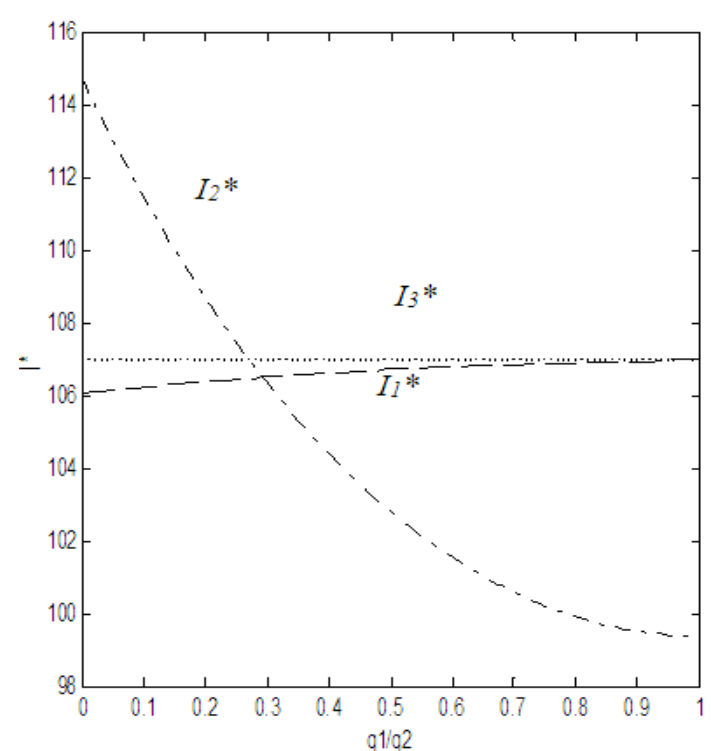

Figure 3: Exponential distribution of stochastic occurrence time with $\lambda=1$ and uniform distribution of stochastic demand.

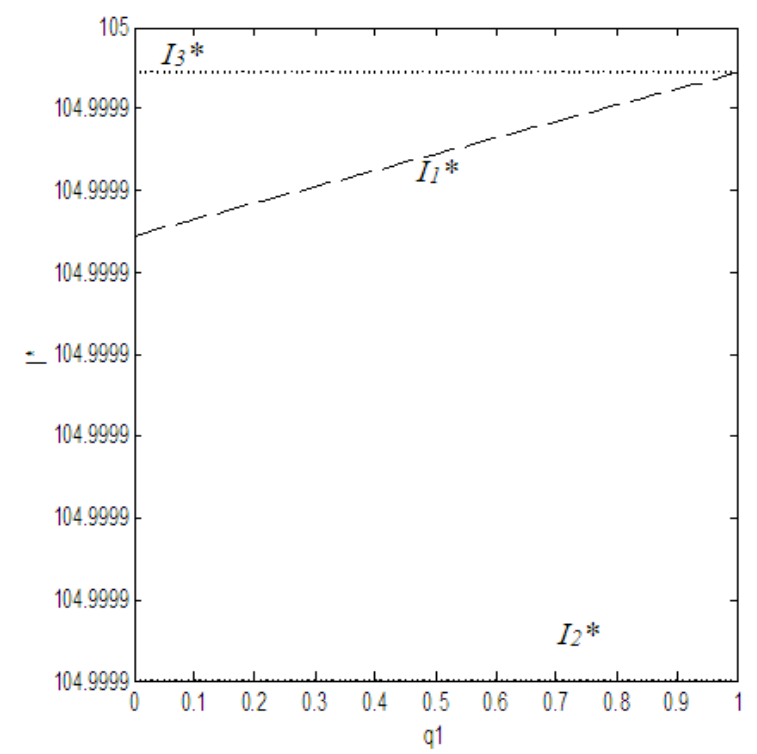

Figure 5: Uniform distribution of stochastic occurrence time and normal distribution of stochastic demand with $\mu=105, \sigma=1 / 105^{2}$.

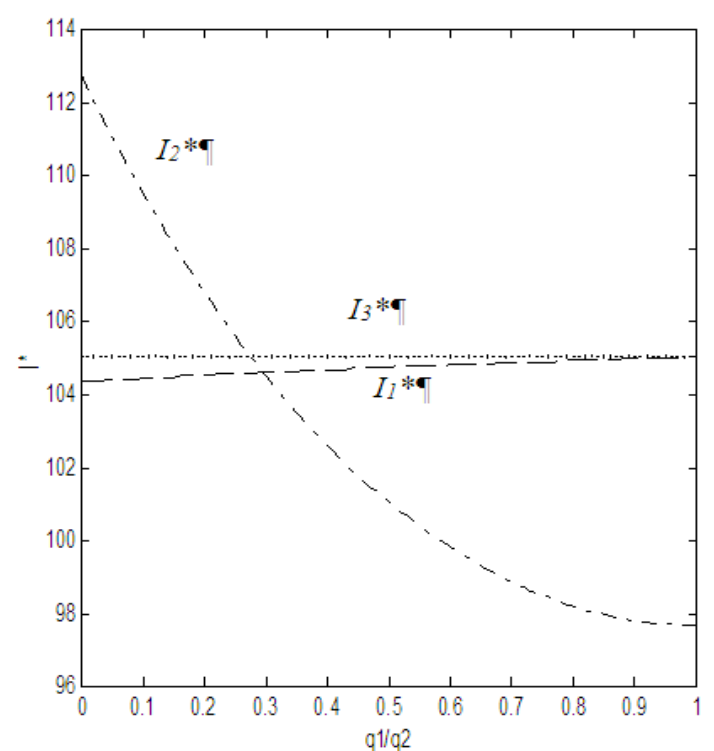

Figure 4: Exponential distribution of stochastic occurrence time with $\lambda=1 / 2$ and uniform distribution of stochastic demand.

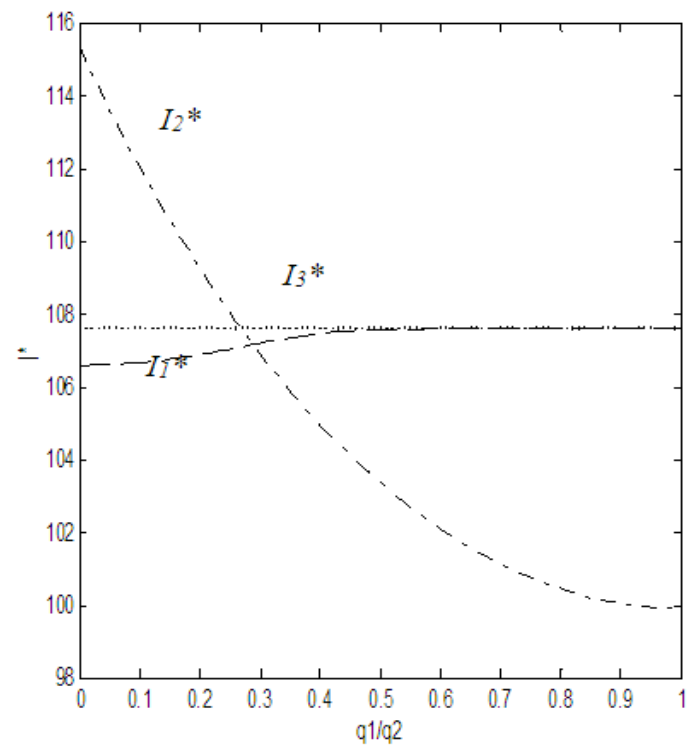

Figure 6: Normal distribution of stochastic occurrence time with $\mu=1 / 2, \sigma=1 / 4$ and uniform distribution of stochastic demand. 
The results in Figs. 1 to 6 show that different stochastic distribution leads to different inventory strategies and the service levels are more likely to be enhanced with replacement strategy when stochastic demand conforms to normal distribution. $\lambda$ represents the frequency within per unit time and the large value of $\lambda$ means the high probability of civil aviation accident, leading to airport reserves more emergency supplies to deal with emergency and risk. Furthermore, the effect of distributed function in stochastic occurrence time is not so great comparatively.

\section{CONCLUSIONS}

The paper studies an inventory control system with stochastic accident occurrence time and short lifetime of perishable emergency supplies, then first proposes two stochastic models with replacement strategy based on remaining lifetime and remaining quantity of emergency supplies respectively at the end of emergency preparation phase.

The effectiveness of replacement strategy is not only correlated to unit replacement cost, but also to the value of replacement ratio. The accident occurrence time uncertainty and limited warehousing lifetime lower the optimal inventory level, and simulation results show that considering occurrence time uncertainty and limited warehousing time can reduce total costs by $51 \%$ though reduce service level by $4.3 \%$. Simulation results demonstrate that the distribution interval affects optimal solutions, and that different distributed functions in both occurrence time and demand lead to different decisions. Further, solving a replacement model based on remaining lifetime can obtain more reliable solutions.

Our work helps enrich researches on risk management in theory and provide advice and suggestions for airport in practice. However, there is much future work to perfect our research content. Obviously, the network designs and supply chain management between airports and suppliers should be studied deeply. Then, a game model should be proposed between airports, government, society and companies for coordination mechanism. Thirdly, a multi-stage stochastic programming or dynamic planning should be researched on emergency supplies inventory management.

\section{ACKNOWLEDGEMENTS}

This study is supported by the National Nature Science Foundation of China (NSFC) (Grant no. 71572096, 71373188, U1333115).

\section{REFERENCES}

[1] Mete, H. O.; Zabinsky, Z. B. (2010). Stochastic optimization of medical supply location and distribution in disaster management, International Journal of Production Economics, Vol. 126, No. 1, 76-84, doi:10.1016/j.ijpe.2009.10.004

[2] Whybark, D. C. (2007). Issues in managing disaster relief inventories, International Journal of Production Economics, Vol. 108, No. 1-2, 228-235, doi:10.1016/j.ijpe.2006.12.012

[3] Tang, M.; Gong, D.; Liu, S.; Zhang, H. (2016). Applying multi-phase particle swarm optimization to solve bulk cargo port scheduling problem, Advances in Production Engineering \& Management, Vol. 11, No. 4, 299-310, doi:10.14743/apem2016.4.228

[4] Yu, G. D. (2016). Modelling for emergency manufacturing resources schedule to unexpected events, International Journal of Simulation Modelling, Vol. 15, No. 2, 313-326, doi:10.2507/IJSIMM15(2)10.348

[5] Taskin, S.; Lodree Jr., E. J. (2010). Inventory decisions for emergency supplies based on hurricane count predictions, International Journal of Production Economics, Vol. 126, No. 1, 66-75, doi:10.1016/j.ijpe.2009.10.008 
[6] Rawls, C. G.; Turnquist, M. A. (2010). Pre-positioning of emergency supplies for disaster response, Transportation Research Part B: Methodological, Vol. 44, No. 4, 521-534, doi: $10.1016 /$ j.trb.2009.08.003

[7] Li, W.; Kou, G.; Ergu, D.-J. (2012). Analysis of emergency resources inventory level based on demand and situation, Chinese Journal of Management Science, Vol. 20, No. S1, 279-283

[8] Kunz, N.; Reiner, G.; Gold, S. (2014). Investing in disaster management capabilities versus pre-positioning inventory: A new approach to disaster preparedness, International Journal of Production Economics, Vol. 157, 261-272, doi:10.1016/j.ijpe.2013.11.002

[9] Das, R.; Hanaoka, S. (2014). Relief inventory modelling with stochastic lead-time and demand, European Journal of Operational Research, Vol. 235, No. 3, 616-623, doi:10.1016/ j.ejor.2013.12.042

[10] Bozorgi-Amiri, A.; Jabalameli, M. S.; Mirzapour Al-e-Hashem, S. M. J. (2013). A multi-objective robust stochastic programming model for disaster relief logistics under uncertainty, OR Spectrum, Vol. 35, No. 4, 905-933. doi:10.1007/s00291-011-0268-x

[11] Pradhananga, R.; Mutlu, F.; Pokharel, S.; Holguin-Veras, J.; Seth, D. (2016). An integrated resource allocation and distribution model for pre-disaster planning, Computers \& Industrial Engineering, Vol. 91, 229-238, doi:10.1016/j.cie.2015.11.010

[12] Davis, L. B.; Samanlioglu, F.; Qu, X.-L.; Root, S. (2013). Inventory planning and coordination in disaster relief efforts, International Journal of Production Economics, Vol. 141, No. 2, 561-573, doi:10.1016/j.ijpe.2012.09.012

[13] Paul, J. A.; MacDonald, L. (2016). Optimal location, capacity and timing of stockpiles for improved hurricane preparedness, International Journal of Production Economics, Vol. 174, 11-28, doi:10.1016/j.jjpe.2016.01.006

[14] Pan, W.; Guo, Y.; Liu, S.; Liao, S. J. (2015). Research on inventory model of airport emergency supplies with random occurrence time, Management Review, Vol. 27, No. 10, 195-203

[15] Liang, Y.; Qiao, P. L.; Luo, Z. Y.; Song, L. L. (2016). Constrained stochastic joint replenishment problem with option contracts in spare parts remanufacturing supply chain, International Journal of Simulation Modelling, Vol. 15, No. 3, 553-565, doi:10.2507/IJSIMM15(3)CO13

[16] Hu, Z.-H.; Sheu, J.-B. (2013). Post-disaster debris reverse logistics management under psychological cost minimization, Transportation Research Part B: Methodological, Vol. 55, 118-141, doi:10.1016/j.trb.2013.05.010

[17] Toso, E. A. V.; Alem, D. (2014). Effective location models for sorting recyclables in public management, European Journal of Operational Research, Vol. 234, No. 3, 839-860, doi:10.1016/j.ejor.2013.10.035

[18] Ying, X.; Breen, L.; Cherrett, T.; Zheng, D.-C.; Allen, C. J. (2016). An exploratory study of reverse exchange systems used for medical devices in the UK National Health Service (NHS), Supply Chain Management: An International Journal, Vol. 21, No. 2, 194-215, doi:10.1108/SCM-07-2015-0278

[19] Lee, W.-H.; Chiu, T.-F.; Ng, C.-J., Chen, J.-C. (2002). Emergency medical preparedness and response to a Singapore airliner crash, Academic Emergency Medicine, Vol. 9, No. 3, 194-198, doi:10.1197/aemj.9.3.194

[20] Pan, W.; Guo, Y. (2015). Bargaining game of compensation in the major civil aviation accidents, Journal of Wuhan University of Technology (Social Sciences Edition), Vol. 28, No. 5, 843-849

[21] Cheng, Y.-H.; Liang, Z.-X. (2014). A strategic planning model for the railway system accident rescue problem, Transportation Research Part E: Logistics and Transportation Review, Vol. 69, 75-96, doi:10.1016/j.tre.2014.06.005

[22] Duran, S.; Gutierrez, M. A.; Keskinocak, P. (2011). Pre-positioning of emergency items for CARE International, Interfaces, Vol. 41, No. 3, 223-237, doi:10.1287/inte.1100.0526

[23] Hsu, V. N. (2000). Dynamic economic lot size model with perishable inventory, Management Science, Vol. 46, No. 8, 1159-1169, doi:10.1287/mnsc.46.8.1159.12021

[24] Hua, G. W.; Cheng, T. C. E.; Zhang, Y.; Zhang, J. L.; Wang, S. Y. (2016). Carbon-constrained perishable inventory management with freshness-dependent demand, International Journal of Simulation Modelling, Vol. 15, No. 3, 542-552, doi:10.2507/IJSIMM15(3)CO12 
[25] Qi, Y.; Tang, M.; Zhang, M. (2014). Mass customization in flat organization: The mediating role of supply chain planning and corporation coordination, Journal of Applied Research and Technology, Vol. 12, No. 2, 171-181, doi:10.1016/S1665-6423(14)72333-8

[26] Luscombe, R.; Kozan, E. (2016). Dynamic resource allocation to improve emergency department efficiency in real time, European Journal of Operational Research, Vol. 255, No. 2, 593-603, doi:10.1016/j.ejor.2016.05.039

[27] Guide Jr., V. D. R.; Souza, G. C.; Van Wassenhove, L. N.; Blackburn, J. D. (2006). Time value of commercial product returns, Management Science, Vol. 52, No. 8, 1200-1214, doi: $10.1287 / \mathrm{mnsc} .1060 .0522$

[28] Shyur, H.-J. (2008). A quantitative model for aviation safety risk assessment, Computers \& Industrial Engineering, Vol. 54, No. 1, 34-44, doi:10.1016/j.cie.2007.06.032

[29] Skorupski, J. (2016). The simulation-fuzzy method of assessing the risk of air traffic accidents using the fuzzy risk matrix, Safety Science, Vol. 88, 76-87, doi:10.1016/j.ssci.2016.04.025

[30] Sun, R. S.; Meng, L. H. (2013). Time distribution of aviation accident, Journal of Transport Information and Safety, Vol. 31, No. 2, 83-87 